\title{
OFFEQ S: WIDE-FIELD UV IMAGING WITH THE ISRAELI SCIENTIFIC SATELLITE
}

\author{
N. Brosch \\ The Wise Observatory \\ Tel Aviv University \\ Tel Aviv 69978, Israel
}

\begin{abstract}
The Israeli Space Agency approved funds for a Phase A study of a scientific satellite to be orbited by a national launcher. Our proposal of an imager in the UV with a $2^{\circ}$ field of view (FOV) is one of the two approved for Phase A. We discuss properties of the orbital platform and requirements for the scientific payload. Some scientific programs for the mission are detailed.
\end{abstract}

\section{OVERVIEW}

The first Israeli satellite, OFFEQ 1, was launched from Israel on September 19, 1988, and orbited till mid-January 1989. The satellite was spin-stabilized and was controlled from a ground station in Israel. Even before its launch, the Israeli Space Agency called for proposals of experiments to be placed on a low-Earth-orbit (LEO) national satellite. Among many proposals from the Israeli scientific community, ours is one of the two selected for Phase A study. The satellite will be a version of the OFFEQ series.

The baseline characteristics of OFFEQ $S$ (the scientific platform) are similar to those of OFFEQ 1, with some important differences that reflect the capabilities of the scientific mission. OFFEQ $S$ is an octagonal cone with a base diameter of $120 \mathrm{~cm}$ and a height of $230 \mathrm{~cm}$. It will be stabilized on three axes with reaction wheels, gyros, sun and horizon sensors, and magnetometer (no star sensors). It will also be equipped with hydrazine motors for reaction wheel discharge and orbit adjustment. Finally, it will be placed on a nominal circular orbit at 400 $\mathrm{km}$.

The constraints imposed on the scientific payloads, as of now, are mass of $20 \mathrm{~kg}$ or less, power consumption of $20 \mathrm{~W}$ or less, and cylindrical volume with $50 \mathrm{~cm}$ diameter and $150 \mathrm{~cm}$ height. The acquisition of target by the orbital platform will be to within $0: 1$, and the position keeping will show jitter of 4-5" and drift of 0.1 per hour. The command telemetry will be only from the Israeli ground station.

The Tel Aviv Astronomy group proposed to orbit a wide-field imager in the ultraviolet (UV) region from about 1400 to $2800 \AA$. The sky is darkest in this segment of the electromagnetic spectrum, where the zodiacal light contribution decreases toward shorter $\lambda \lambda$, and the scattered galactic light starts increasing. Moreover, we felt that the techniques required for UV imaging in this region are not too different from those of optical imaging, in which we acquired considerable experience at the Wise Observatory.

The allowed size and mass of the payload imply that only a modest imaging system will be orbited. The pointing accuracy of the platform, compounded with the fact that observations would have to be preplanned, constrain us to propose relatively small telescopes with reasonably wide fields (so as not to miss our targets). It appears that very important scientific experiments may be performed with a wide-angle imager. 
The stability of the platform requires either a compensating mechanism, to prevent the drift from smearing the images, such as an articulated secondary mirror, or relatively rapid readout of the images, with subsequent on-board or on-ground combination into a longer exposure. The jitter of the carrier platform implies that, at best, our images will be of 4 " FWHM, similar to seeing effects of ground-based telescopes at sub-average locations.

\section{FUTURE RESEARCH IN THE UV}

Satellites provided many years' worth of aperture photometry and spectroscopy, but imaging has been done mainly by rocket experiments and amounts to only a few hours in space. The future of UV imaging astronomy lies mainly with the Hubble Space Telescope. This is planned to be orbited in 1990 and is an LEO instrument with $2.4 \mathrm{~m}$ aperture and with a widest FOV of 2.' 4. Another imaging experiment is ASTRO, an STS payload with three approved missions, each a few days' duration.

ASTRO is the only future payload that has some wide-field imaging capabilities. These come from the UIT segment, a $38 \mathrm{~cm}$ diameter f/9 telescope with a $40^{\prime}$ FOV and 2 "angular resolution, developed at Goddard Space Flight Center. In a $30 \mathrm{~min}$ exposure, the UIT will record a $V=25$ star of $T_{\text {eff }}=30000 \mathrm{~K}$, with a signal-to-noise of 10 , on film carried on-board. The method of recording limits the dynamic range.

\section{UVEX - ISRAELI NATIONAL UV PAYLOAD ON OFFEQ S}

We wish to image an area larger than that to be imaged by the UIT payload on ASTRO, with reasonable angular resolution and with good signal-to-noise. At present we are studying two designs, one with a single telescope with $\sim 50 \mathrm{~cm}$ optics and another with four $\sim 20 \mathrm{~cm}$ telescopes, with better redundancy but smaller collecting area per detector. The detectors under consideration are intensified CCDs, with CsI or CsTe cathodes, fiber-optically coupled to the IT phosphors.

The spectral region of interest is from 1400 to $2900 \AA$, which we want to observe in at least three spectral bands. Nominally, then, we have three spectral regions, each some $500 \AA$ wide. The fourth telescope, if a four-shooter design will be adopted, may image in the entire UV band for the deepest exposures.

Assuming telescopes of $20 \mathrm{~cm}$ diameter, the expected sky photon rate at $2000 \AA$ will be - 2 photons $1000 \mathrm{~s}^{-1}$ exposure $\operatorname{arcsec}^{-1}$, with a $500 \AA$ bandpass. Adopting a prime focus design, with $10^{\prime \prime}$ pixels, relevant because the coma distortion will be less than $10^{\prime \prime}$ at $1^{\circ}$ off axis, a mirror reflectivity of 0.85 , a transmission of 0.50 for the filters, and a quantum efficiency of 0.1 for the IT, we should detect about 800 events per pixel per $1000 \mathrm{~s}$ exposure from the sky alone.

An extended object can usually be detected from the ground when its surface brightness is only $1 \%$ that of the sky. Assuming similar capability for UVEX, this means that it should be possible to detect features at $31 \mathrm{mag} \operatorname{arcsec}^{-2}$ at $2000 \AA$. This is probably too optimistic, considering the intrinsically low photon counting rate with a small telescope.

We will dedicate large parts of observing time to deep imaging of selected regions of sky. Among those there are the two galactic caps to $|b|>80^{\circ}$, the Virgo cluster, and Local Group galaxies. As an example, we describe the Virgo cluster mapping program.

The Virgo cluster is the nearest rich cluster of galaxies. Its population of galaxies was cataloged in the visible by Binggeli and collaborators. The region covered by the Binggeli 
survey was from $12^{h}$ to $13^{h}$ in right ascension and from $0^{\circ}$ to $+20^{\circ}$ in declination. This is about $300 \mathrm{deg}^{-2}$. To cover the same area, we require less than 100 . This can be completed in six days, using only the dark part of the orbit. The planned lifetime of the satellite, one year with $90 \%$ confidence, allows for about 10,000 pointings (two per orbit). Thus to map entirely the Virgo cluster, we must dedicate $1 \%$ of its time to this task. A similar project with the Hubble Space Telescope would require about 160,000 observations with the Wide Field Camera.

The output of this mapping program will be the surface photometry in three UV bands of more than 2000 galaxies of various morphological types. This will serve for studies of the hot stellar populations in galaxies, the UV excesses in central regions of elliptical galaxies, population synthesis of $\mathrm{S}$ and $\mathrm{E}$ galaxies, characterizing interacting galaxy systems and spiral structure, as well as the ISM in galaxies. In addition, searches will be made for low-surface brightness galaxies (faint Im's, Malin-1 types, etc.) and faint circumgalactic material.

The capabilities of the instrument to measure the UV background are considerable, because of the possibility of separating foreground stars and resolved galaxies from true sky background. In particular, the survey of the Virgo cluster shall yield an estimate of the intracluster light. Similarly, the polar caps survey will concentrate on regions with the lowest (solar system-induced) surface brightness.

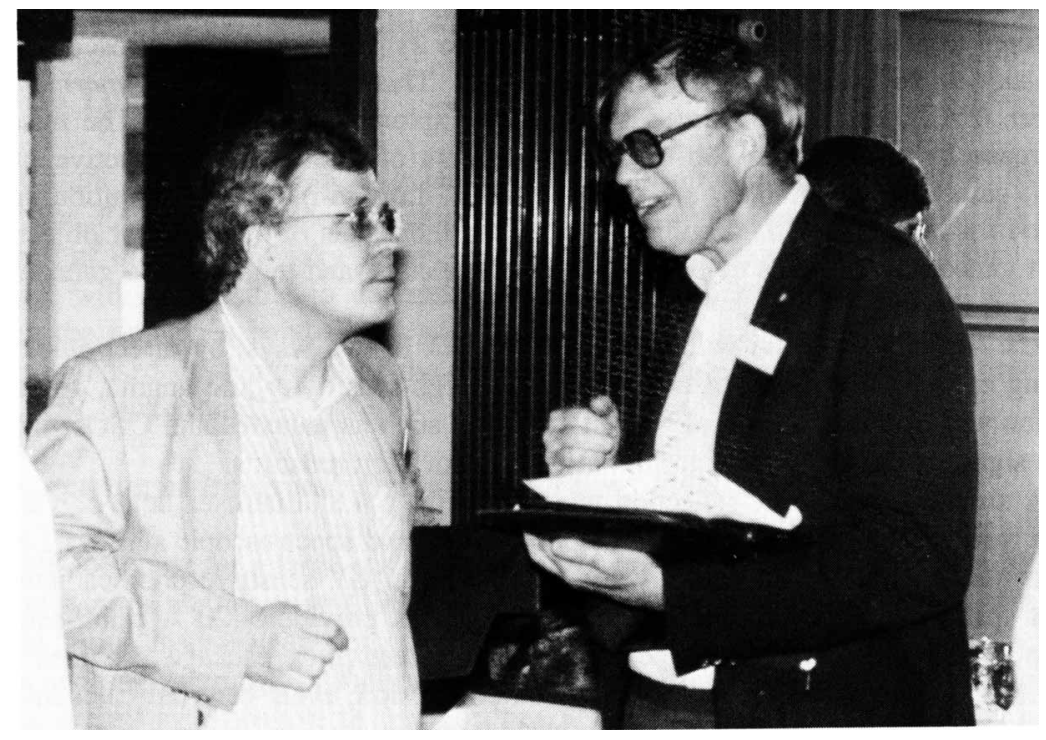

Kalevi Mattila and Stuart Bowyer 